BNL 19008

EP\&S 74-3

BROOKHAVEN NAT IONAL LABORATORY

Associated Universities, Inc. Upton, New York

ACCELERATOR DEPARTMENT

Informal Report

INIT LAT ION OF THERMONUCLEAR DETONATIONS

A.W. Maschke

June 5, 1974

ABSTRACT

In the following note we derive scoe simple relations describing $a$ thenwonuclear detonation in a deuterium-tritium mixture. We propose an 1gnition system which consists of a storage ring, (or set of them) in which a bunched beam is stacked up to the transverse space charge limit. The paiticles are then extracted and focused to an appropriate spot. It is this sudden heating which then initiates the thezmonuclear shock wave in the fuel. The practical problems of storing and focusing of the beam and containment of the detonation are not treated in this note, but will be dealt with in a later paper.

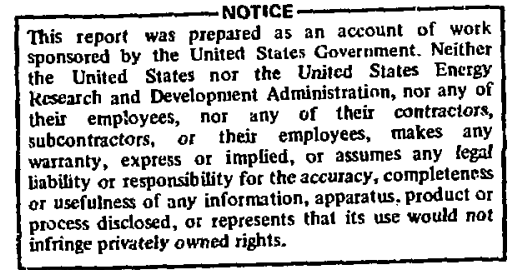

\title{
NOTICE
}

This report was prepared as an account of work sponsered by the United States Goverment. Neither the Unfted States nor the United States Atomic Energy Cormission, nor any of their employees, nor any of their contractors, subcontractors, or their employees, makes any warranty, express or implied, or assumes any legal liability or responsibility for the accuracy, completeness or usefulness of any information, apparatus, produc or process disclosed, or represents that its use would not infringe privately owned rights. 


\section{Initiation of Thermonuclear Detonations}

I. We consider a cold plasma, of density $\rho_{0}$, and a detonation wave moving at velocity $U$. Behind the detonation wave we have a density $\rho_{1}$, a velocfty $\mu$, pressure $r$, and internal specific energy $\triangle E$. The conservation of mass, momentum, and energy give rise to the three Rankine-Hugoniot shock relations.

$$
\begin{aligned}
& \rho_{0} U=\rho_{1}(U-\mu) \\
& P=\rho_{0} U \mu \\
& \Delta E=\frac{1}{2} \mu^{2}
\end{aligned}
$$

If we take the plasma to obey the perfect gas law, we have the equation of state.

$$
P=\frac{2}{3} \quad n_{1} T=\rho_{1} v_{t h}^{2}
$$

where $n_{1} \equiv \rho_{1} / M$, and $T \equiv 3 / 2 M V_{t h}$, and $M$ is the mass of the ions. We can now solve these equations as follows:

$$
\begin{aligned}
& \rho_{2} / \rho_{0}=4 \\
& D=\frac{4}{\sqrt{3}} v_{t h}
\end{aligned}
$$

We need one more equation to solve for $v_{t h}$, or what is the same thing, $T$. We obtain this relation from the following considerations. A wave is moving with velocity $U$ through a medium of density $p_{o}$, and heating it to temperature 7 . Therefore, there must be a power source which provides power at the following rate:

$$
P_{\text {in }}=U n_{0} T \text { watts/meter }{ }^{2}
$$

Using our previous relations we can rewrite this as follows:

$$
P_{\text {in }}=\frac{4}{3} r \frac{2}{M} n_{0} T^{3 / a}
$$

Now the power source is the fusion reaction, and the shock wave is propogated by the $3.5 \mathrm{MeV} \alpha$-particles losing energy in the plasma. The rate of production of $\alpha$ particles/unit volume is $R=\frac{n}{\frac{\lambda}{4}}\langle\sigma v\rangle$, where $\langle\sigma v\rangle$ is a function of temperature. Since the $\alpha$ particles have a range, $\lambda_{\alpha}$, only the $\alpha$ particles within this distance will be able to supply heat to the shock front. Taking $1 / 6$ for the geometry factor, we obtain: 


$$
P_{\text {out }}=\frac{E_{0} n^{2}}{24}\langle\sigma v\rangle \lambda_{\alpha}
$$

Fihere $\mathrm{E}_{\alpha}$ is the energy of the $\alpha$ particle.

The range of $\alpha$ particles in a plasma is a function of temperature, and the relation 18 given $48: \lambda_{\alpha}=.78 \times 10^{49} \mathrm{~T}^{3} \mathrm{n}_{\mathrm{l}}^{-1}$. Equating $\mathrm{P}_{\text {in }}$ co $P_{\text {out }}$, we have our $\hat{x}$ inal relation:

$$
\frac{4}{3}: \sqrt{\frac{2}{M}}=.78 \times 10^{49} \frac{E}{6}\langle\sigma v\rangle
$$

This detexwines a value of $\langle o v\rangle$ which is required in order to support a detonation in a Dr mixture. Note that the density does not cone into this relation. We obtain $\langle\sigma v\rangle \approx 3.8 \times 10^{-23} .^{3}$ This corresponde to a temperature of about $7 \mathrm{KeV}$. If our estimates were wrong by a factor of two, the required tewperature would rlse to $9 \mathrm{Kev}$. Becsuse we did not cake radiation losses into sccounc in equation 4 , it is likely that the higher estinate is more appropriate. We aloo did not take the heating due to the $14 \mathrm{Mey}$ neutrons into eccount. This is reasonable for considering the condition for initiating the detonation. After the detonation has proceeded for a distance like the neutron mean free path, we have a large additional source of power. Therefore temperatures in the core would be expected to rise, and other fuels could be burned.

II. We have sean in the previous section that a cemperature of about $10 \mathrm{KeV}$ is required to propogate a detonation wave in a D-T mixture. In this section we will estimate the amount of energy input required to initiate a detonation. The critical mass is on the order of a spere of radius $\lambda_{\alpha}$, the range of the 3.5 Mev $\alpha$-particle. Therefore we have:

$$
M_{\text {crit }} \geqslant-\frac{4 \pi}{3} \rho \lambda_{\alpha}^{3}
$$

We define a compression factor $\alpha$, by the relation $p=\rho_{0} \alpha$. We will take $P_{0}$ to be the density of a liguld D-T mixture, or more explicit ly, correaponding to an electlon density of $5 \times 10^{28}$ meter ${ }^{-3}$. At the 1 gnition -emperature we have $\lambda_{\alpha}=10^{-2} \alpha^{-1}$, wo the critical mase becones $M_{\text {crit }}=(4 \pi / 3) 10^{-B} \rho_{0} \alpha^{-2}$. The energy necessary to bring this mass up to the critical temperature then 1s: 


$$
E_{\text {crit }}=600 / \alpha^{2} \text { MJoules }
$$

Several points are worthy of note here. One is the strong dependence upon $\alpha$. The laser fusion scheme make use of this in the extreme, with $\alpha=10^{4}$. The other point relates to our assumption about the $\alpha$ particle range. In the case that we have high magnetic fields present, the range might be substantially shorter. Since this factor enters cubically ints the equation, one might indeed not expect equation 5 to provide more than a rough estimate.

III. The importance of initiating a detonation in order to achieve high energy gains can be seen from the following considerations. We can define a reaction $t i m e t_{\text {reaction }}^{-1}=n\langle o v\rangle$. For an inertially confined plasma, the expansion time for a critical mass is $t_{\text {exp }}=\lambda_{\alpha} / V_{t h}$. If we put in * $10 \mathrm{Kev}$ to heat the plasme, and obtain $17000 \mathrm{kev}$ from each fusion reaction, then the net energy yield is given by:

$$
Y_{\text {crit }}=1700\langle o v\rangle \cdot \frac{n \lambda_{\alpha}}{v_{t h}} \approx 100
$$

Note that this is independent of $\alpha$. Such a smell yield ratio makes it ifficult to achieve a net useful energy gain. In order to break even one must consider the heating efficiency, $T_{h}$, the boiler/turbine efficiency, $\eta_{t h}$, which is usually around . 35, and the beam energy/input energy for the trigger device, $T_{i b}$. If a detonation is started, however, the yield is virtually unlimited. The problem becomes one of contairment for the explosion.

IV. So far we have only discussed the energy requirements for initiating a thermonuclear reaction. In this section we will examine the power requirements. In order to get some feeling for the times that are involved we write down the following time constants:

$$
\begin{aligned}
t_{\text {Bremstrahlung }} & =\frac{2 \times 10^{-8}}{\alpha} \\
t_{\text {reaction }} & =\frac{29 \times 10^{-8}}{\alpha} \\
t_{\text {conduction }} & =\frac{1.33 \times 10^{-8}}{\alpha} \\
t_{\text {expansion }} & =\frac{1.8 \times 10^{-8}}{\alpha}
\end{aligned}
$$


A word of caution is required for the time constant for thermal conductivity. This depends upon the electrical conductivity, and ancomolous resistivity due to turbulence effects may increase thio substantially. On the other hand, turbulent mase transport may also rend to enhance the heat conduction. Experimental results are needed here.

Uaing equacion 5 for the crittcal energy, we obtain an approximate value of the power requirement to be $P_{c} \geqslant 6 \times 10^{26} \alpha^{-2}$ watts.

v. Now we will consider energy deposition in the plasma by relativiatic charged particles. A particle with charge 2 will lose $.6 \mathrm{z}^{\mathrm{a}}$ Kev in transversing the diameter of a critical mass of D-T. Since the particles we will be conslderiag, wil! have much higher energies than this, they will generally be capable of substantial penetration. In what follows, we will always aseume that we are dealing with a cylindrically aymetrical distribution, with the energy deposition along the axis. The number of charge particles required for 1 gnition 19 given by:

$$
N_{c}=\frac{6 \times 10^{21}}{2^{2}}
$$

Two extremes are of interent in this regard. Electrons are attractive because of their low mass. This gives rise to radiation damping in a storage ring, and to palr production and Bremstrahlung in its interactions. By "ahowering" the electrons before entering the Dr region, substantial particle multiplication can be achieved. On the other hand, high 2 lons, like Uranium, aze required in much fewer numbers. Equation 6 exhibits the basio design problem. It is to devise a means of achieving high compressions, and to obtain a large number of particles in a short time.

The instantanecus current required in the charged particle beam is given by:

$$
1_{c}=\frac{z e N c}{=}=\frac{z}{z^{3} \alpha} \times 10^{21}
$$

where $z$ refers to the charge of the particle before it is etripped in the energy lose procese. For the case of electrons, this must be corrected by the particle nultiplication factor $\mathrm{K}$, which could be as high as 100 . 
VI. So far we have specifled the Inatancaneous curzenc, hunch widch, and particle numbers needed for Ignicion of an "unconfined" yutem. Thear ull critically depend on $a$. In this eceten we will derive a relacton independent of a which will tell us wore about the properties of the charged particle teams. He consider the traneverso space charge lfoic for an unneutralized beam in a circular strong focusing storage device. In the limit of tight bunches and large apertures, one can neglect che effects of image forces. In this case the space charge limiting current can be written: ${ }^{4}$

$$
I_{\max }=.8 \times 10^{7} \in \frac{Y^{3}}{R} \frac{A}{z}
$$

Here we have taken $\pi$ to be the crangverse emitcance, and acsued a tune shift of .25. $R$ is the sverage redius of the storage ring. Clearly, we would like $\varepsilon$ to be large. However, stnce we must focus this beam onto a spot, there is a limit to this. Since the radius of the spot ause be no larger than $10^{-\%} / 0$, and if we assume . I radkan for the grcateat angular divergence which is practical, we obtain $c: 10^{-4} / \mathrm{f}$. Insercing this inco 8, and equating with 7, wa obtain the collowing rewult, which is independent of $x$ :

$$
\frac{A Y^{3}}{R}=1.25 \times 10^{-1}\left(\frac{z}{2}\right)^{2} K^{-1}
$$

For electrons, this comes out to be $\gamma^{3} / 8=2.3 \times 10^{10} K^{-1}$. If we take $\mathrm{A}$ to be 100 meters, and $K=100$ we obtain $\gamma=2800$, corresponding to about $1.5 \mathrm{Gev}$. For $U$ lons, we use the relation $B R=8 / 2 c$, and express $r$ at $a$ function of the average magnete field. We obtain:

$$
\begin{aligned}
& \gamma=66 \frac{\sqrt{2}}{B} \\
& B R=\frac{4.76 \times 10^{4}}{V 22}
\end{aligned}
$$

Since BR is a quancicy one wishes to keep small, one vante high $B$ and high $z$. Taking $B: 3$, and $z=92$, we obtain $R=939$ weters, $y=366$. Thia is equivalent to a 900 Gev proton storage ring.

VII. It must be emphasized that most of the forcgoing relaticns are based upon an assumption that magnetic confinement did not effect our eatimate of $\alpha^{\cdot}$ Furthermore, the magnetic fields will act to reta: $t$ the expansion 
6 the plaow accross the field lines. The magnetic rigidity of 3.5 Mev a particle to .26 T-m. The fleld aecociaced with line current io given by:

$$
\text { B }-2 \times 10^{-7} \frac{1}{\mathrm{r}}
$$

The condition that we have enough current to bend the $\alpha$ particle is then:

$$
1 \text { - } 5 \times 10^{8} \text { anps }
$$

Th1. condition to ecsentially Independent of the else of the current source. Hence it does not depend upon $\alpha$. Compring this result with that of equation 7 , we see that in fact the magnetic fieldu way play a large role Indeed. The eotabliohnent of large magnetic fielde in a dense hot plaema 10. aubject of coniderable experinental and theoretical Intereat at the present time.

Diotribetion: 82

\section{REFERENCES}

1. W.C. Davis, LA-DC-72-214, Detonation Phencmenon.

2. K.A. Bruckner and H. Bryak, J. Plaena Phys1ce 10, 141 (1973).

3. J.L. Tuck, Nuclear Fusion 1, 201 (1961), Themonuclear Reaction Ratee.

4. L. J. Lalett, Proc. of the 1963 surer Study on storage Ringe at Super High Energies (J.W. Bittner, ed.), BuL Rejort 7534, p. 324 (1963). 